\title{
Editorial corner - a personal view Are we closer to a solution for the plastics waste disaster which faces the earth?
}

\author{
A. S. Luyt* \\ Center for Advanced Materials, Qatar University, Doha, Qatar
}

In my previous 'Editorial corner' contribution I discussed the question whether biodegradable polymers are the solution to the world's plastics waste problem, and I concluded that scientists and policy makers are faced with two major challenges: the invention/design of completely biodegradable polymers/ blends/(nano)composites that can replace all the non-biodegradable polymers currently in use, and the recovery of all the biodegradable polymers that reached their end-of-use, and dispose them in suitable commercial composting sites for fast and effective biodegradation.

Now, two years and a significant number of publications and patents later, we are still not much closer to a solution, and plastics, despite their usefulness in a large variety of applications, are slowly but surely destroying our environment. The biggest culprits are plastic shopping bags and disposable plastic items used in the food industry. The fact that these items tend to be manufactured from 'biodegradable' polymers, may reduce their impact on the environment, but there are other items made from completely nonbiodegradable polymers that are used outdoors and replaced very frequently because of partial UV/heat initiated degradation which is the result of the severe weather conditions in certain parts of the world. An example is the kilometers of polyethylene irrigation pipes used above ground in parks and gardens in some countries in the Middle East to create a green environment in desert cities. Huge quantities of these pipes, and other outdoor used plastic items, are also stored in warehouses where they constitute a fire hazard because of their flammability.

It would therefore make sense to carry on with research on biodegradable polymers, composites and/or nanocomposites that can replace current nonbiodegradable plastics, but there should also be a certain amount of research into increasing the lifetime of plastics for outdoor use. Relatively cheap (and preferably 'green') combinations of UV/heat stabilization and fire retardant additives, that will significantly increase the lifetime of non-biodegradable plastics designed for long-term outdoor use and that will also reduce their flammability making them safe for indoor storage, will certainly help to achieve these goals.

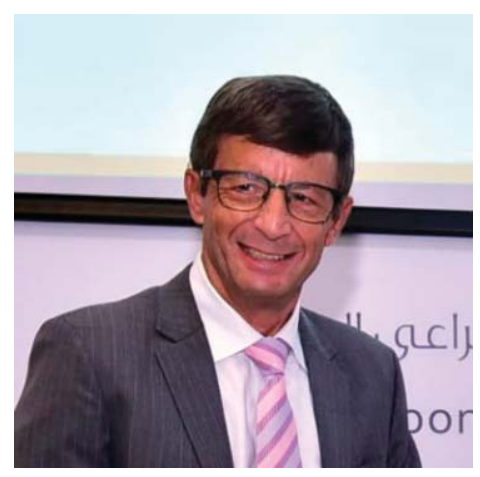

Prof. Dr. Adriaan Stephanus Luyt Member of the International Advisory Board 